\title{
Aumentar el consumo responsable de agua mediante la exposición a consecuencias futuras con realidad virtual*
}

Increasing the Responsible Consumption of Water with Presentations of Virtual Reality Showing the Future Consequences

Elena Ochoa-Martinez

Centre de Teräpia Interfamiliar, España

DOI: https://doi.org/10.11144/Javeriana.ayd23-45.acra

ORCID: http://orcid.org/0000-0003-4641-1932

Recepción: 02 Febrero 2019

Sergio Pérez-Gonzaga a

Universidad Miguel Hernández de Elche, España

Aceptación: 10 Abril 2019

sperezgonzaga@gmail.com

ORCID: http://orcid.org/0000-0001-5187-6895

Publicación: 30 Diciembre 2019

\section{Resumen:}

Se ha demostrado que un aumento de la concienciación influye sobre el uso responsable del agua, sin embargo, las campañas de concienciación tienen un efecto de limitada duración en el tiempo y se observa que buenas actitudes hacia el medio ambiente no siempre acompañan una conducta positiva. En este trabajo se propone influir en las actitudes del consumidor, de manera que tenga una influencia positiva en un consumo responsable; para ello, se tendrá en cuenta el descuento de la demora, que sería el grado en que una consecuencia futura se valora en el presente de forma devaluada por su lejanía en el tiempo. Basándonos, por un lado, en estudios sobre las actitudes hacia el ahorro de agua y su influencia en el consumo, y por otro lado, en el efecto que ha tenido la influencia en el descuento de la demora sobre el comportamiento de las personas en otras conductas problemáticas. Se propone una campaña de concienciación de ahorro de agua en el hogar, influyendo sobre el descuento de la demora.

Palabras clave: ahorro de agua, concienciación, manejo responsable de recursos, consecuencias futuras.

\section{Abstract:}

It has been shown that raising awareness influences strongly the responsible use of water. However, awareness campaigns have a short-lasting effect and it has been noted that environmentally-friendly intentions are not always accompanied by positive behaviors. This work aims to influence the consumer's attitudes, so that to induce a positive behavior of responsible consumption. This effort requires taking into consideration the discount based on the delay, i.e., to what extent a future consequence is deemed in the present as devaluated due to the distance in the time. Using studies dealing with the attitudes before the water saving and its influence on the consumption and, on the other hand, the effect of such influence on the delay-based discount regarding the people's behavior when facing other problems, this work proposes an awareness campaign for saving water at home, in order to influence on the delay-based discount.

Keywords: water saving, awareness-raising, resource responsible management, future consequences.

\section{Introducción}

Se ha demostrado que un aumento de la concienciación influye sobre el uso responsable del agua (Willis, Stewart, Panuwatwanich, Williams y Hollingsworth, 2011), sin embargo, las campañas de concienciación tienen un efecto de limitada duración en el tiempo (Stavenhagen, Buurman y Tortajada, 2018; Inman y Jeffrey, 2006). También se han analizado otras medidas como introducir medidores de agua inteligentes o hacer las tarifas más transparentes, mediante una facturación más frecuente y clara, lo cual tiene un efecto en la racionalización del consumo (Stavenhagen et al., 2018; European Enviroment Agency [EEA], 2001; Gaudin, 2006).

Notas de autor

\footnotetext{
a Autor de correspondencia. Correo electrónico: sperezgonzaga@gmail.com
} 
Por otro lado, los hogares de las regiones que han sufrido sequías y restricciones hacen un mejor uso del agua que aquellos que no han estado expuestos a estas (Moglia, Cook y Tapsuwan, 2018), lo cual es indicativo del efecto que tiene sobre el comportamiento de las personas el haber vivido una situación de escasez en primera persona.

La economía conductual busca una mejor explicación de la toma de decisiones de los individuos, integrando el factor psicológico que no ha sido tenido en cuenta en los modelos económicos clásicos que consideraban que los agentes tomaban siempre decisiones racionales. En este trabajo, vamos a utilizar conceptos de la economía comportamental para proponer una nueva forma de influir en las actitudes del consumidor, de manera que tenga una influencia positiva en el consumo responsable.

Muchos programas institucionales, cuya finalidad es educar a los consumidores sobre consumo responsable, se han basado en el supuesto de que un mayor conocimiento y actitudes favorables hacia la conservación del medio ambiente conducen a un consumo más responsable. Sin embargo, estudios como el de Dharshing y Hille (2017) muestran una brecha entre el conocimiento y la actitud hacia el medio ambiente, que pueden ser altos, mientras que la conducta de ahorro no sucede en la medida que era de esperar. Los autores recomiendan un cambio de estos programas de concienciación genéricos por otros más individualizados que influyan en variables comportamentales. También recogen las reticencias de los consumidores en costear a corto plazo medidas de ahorro, a pesar de que en el futuro se haya producido un ahorro total al implantar dichas medidas. En la misma línea, Brick y Lai (2018) revisan la numerosa literatura sobre la brecha actitud-comportamiento y conducen un metaanálisis donde concluyen que cuando la actitud va acompañada de una identidad explícita con la causa el comportamiento pro-causa mejora. La actitud es un constructo psicológico complejo que ha sido estudiado con diferentes modelos, uno de los más influyentes es el multicomponente (Breckler, 1984), donde la actitud es la evaluación de un objeto que tiene tres componentes diferenciados: el cognitivo (donde entraría la información), el afectivo y el conductual.

El objeto de este trabajo es proponer una campaña de concienciación de ahorro de agua en el hogar, influyendo sobre una variable que ha recibido numerosa atención en los últimos tiempos: el descuento de la demora, que sería el grado en que una consecuencia futura se valora en el presente de forma devaluada por su lejanía en el tiempo, y es un concepto fundamental en la llamada economía conductual. Para hacer esta propuesta nos basaremos, por un lado, en la reciente literatura sobre las actitudes hacia el ahorro de agua y conservación del medio ambiente y cómo estas influyen en el consumo; y por el otro lado, en el efecto que ha tenido en diferentes estudios el influir sobre el comportamiento de las personas con el descuento de la demora.

\section{Justificación teórica}

\section{¿Por qué el descuento de la demora?}

En los últimos tiempos se está expandiendo el estudio del descuento de la demora (delay discounting) como una variable importante que influye en numerosas conductas-problema, como las conductas sexuales de riesgo: se ha encontrado que un mayor descuento de la demora se asocia a mayores prácticas sexuales de riesgo (Collado, Johnson, Loya, Johnson y Yi, 2017; Lawyer y Mahoney, 2017). El estudio de Stein, Tegge, Turner y Bickel (2018) ha demostrado que mediante la aplicación del pensamiento episódico futuro se ha logrado reducir tanto el descuento de la demora como la demanda de cigarrillos. También se ha visto en bebedores problemáticos que el PEF reduce el descuento de la demora y que esta reducción aumenta con la repetición de las sesiones (Mellis, Snider, Deshpande, LaConte y Bickel, 2019). También se ha encontrado que este refuerza conductas como drogodependecia, obesidad y juego patológico (Koffarnus, Jarmolowicz, Mueller y Bickel, 2013). 
Se ha investigado el pensamiento episódico futuro (episodic future thinking) como herramienta para reducir el descuento de la demora. El pensamiento episódico futuro se refiere a la capacidad para imaginar experiencias que podrían sucedernos en un futuro. Este fenómeno está abalado por mecanismos neurológicos y cognitivos, así como se ha mostrado su funcionalidad a la hora de obtener beneficios en la toma de decisiones o regulación emocional (Schacter, Benoit y Szpunar, 2017).

La hipótesis en que se basa este trabajo es la siguiente: si el descuento de la demora es una variable importante en el comportamiento responsable del consumidor de agua, exponer a una serie de sujetos a una consecuencia futura de un consumo no responsable reducirá la visión lejana en el tiempo de esa consecuencia y conllevaría un aumento del consumo responsable.

\section{La realidad virtual como medio de intervención}

Usar la realidad virtual es más sencillo y económico que el uso del pensamiento episódico futuro porque, entre otras razones, no requiere entrenamiento en imaginación y se puede aplicar de manera automática. La tecnología que soporta estas técnicas es muy barata y tiene un alto impacto en las personas, que además están muy abiertas a vivir esta nueva experiencia, por lo que resulta más fácil contar con su colaboración. Hay estudios que indican que incluso una breve experiencia con realidad virtual puede ser suficiente para cambiar y mantener el comportamiento real en el mundo físico (Ahn, Fox, Dale y Avant, 2015).

\section{Propuesta de intervención}

\section{Consideraciones previas}

Se ha visto la eficacia del pensamiento episódico futuro como herramienta para reducir el descuento de la demora. La característica fundamental del pensamiento episódico es que ha de estar referido a la propia vida del individuo que lo ejecuta, es decir, que ha de ser una experiencia asumida como parte de la biografía vital de la persona. Por lo tanto, el programa de intervención ha de hacer al individuo vivir el suceso, emulado en primera persona. Para conseguir este efecto, se personalizará el mensaje con datos propios del sujeto, mediante el uso de voz sintética y las imágenes incluirán entornos personalizados, según la localidad de residencia del individuo.

También se ha visto que incluso una breve experiencia puede tener un impacto considerable, por lo que se podrá realizar alguna prueba piloto con apenas coste de producción, y con esos datos preliminares poder tomar la decisión sobre si invertir más en mejorar la experiencia y aplicarla a mayor número de sujetos.

El atractivo de la realidad virtual facilitará mucho la participación voluntaria tanto de los individuos como de las familias. Para trabajar con los individuos y evaluar la intervención no serían recomendables medidas obtenidas por cuestionarios sobre actitudes hacia el consumo responsable del agua, puesto que, como se ha mencionado anteriormente, existe una brecha entre la actitud y el comportamiento. Nos basaremos, entonces, en medidas objetivas de consumo en el hogar, limitándonos a la participación de familias e individuos que vivan solos. Para esta medida objetiva, si no fuera posible incluir las medidas de contadores inteligentes, se usará el consumo en bruto. 


\section{Intervención}

Se propone una campaña con caravanas equipadas con un equipo de realidad virtual, donde se reclute a individuos o familias de la localidad y se les ofrezca vivir la experiencia de viajar al futuro mediante la realidad virtual. Se harán dos grupos: uno control y otro experimental.

Para el grupo experimental, se introducirá en la experiencia una serie de datos personales y se incluirán escenas de la localidad donde residan para poder implicar emocionalmente al sujeto como protagonista de la experiencia, la cual recreará una grave sequía en la región.

La experiencia comenzará indicando a los participantes que se dispone a formar parte de una experiencia piloto en hibernación como parte de investigación en largos viajes espaciales y, a continuación, se le coloca el equipo de realidad virtual.

Un actor, dentro del mundo virtual, despertará al sujeto indicándole que ha pasado hibernando 4 años y este, experimentando con el entorno, irá descubriendo poco a poco que el país se encuentra viviendo una severísima sequía desde hace un año. Se usarán recursos como una televisión donde se emite un informativo con un popular presentador donde narra la situación, el relato de los actores que acompañan al sujeto en el mundo virtual, la visita a lugares donde se reciba de forma impactante el efecto de la sequía y la comprobación virtual en primera persona de la falta de agua. Antes de salir de la experiencia otro actor informará que este escenario se podrá evitar si cada individuo contribuye con un cambio en su comportamiento. Se despide a los participantes con un folleto informativo de medidas a tomar y un cuestionario a cumplimentar con datos de consumo pasados y futuros que deberán remitir más adelante.

El grupo control tan solo vivirá una demostración de un escenario de realidad virtual, donde se le mostrará una serie de imágenes relacionadas con recursos hídricos junto con un mensaje de concienciación de la necesidad del ahorro de recursos (al estilo de las campañas tradicionales), para este grupo, la experiencia será meramente informativa y sin referencias episódicas. A estos también se les dará el folleto informativo y el cuestionario de seguimiento.

\section{Propuesta de validación}

\section{Diseño}

Se define como variable independiente el pasar o no por la sesión de realidad virtual con carácter episódico (variable grupos intersujetos) y como variable dependiente la medida de disminución de consumo (variable intrasujetos).

Para validar esta hipótesis se realizará una investigación cuasi experimental (tabla 1), en la que se utilizará un diseño pretest-postest (medidas repetidas) con el grupo de control (el que realizó la experiencia con realidad virtual sin la referencia episódica).

TABLA 1.

Diseño pretest-postest

\begin{tabular}{|lllll|}
\hline Grupo & Asignación & \multicolumn{3}{l|}{ Secuencia de recolección de datos } \\
\cline { 3 - 5 } & & Pretest & $\begin{array}{l}\text { Sesión con RV + info.I } \\
\text { Exposición episódica }\end{array}$ \\
\hline Experimental & No Aleatoria & $\mathrm{O}_{1}$ & $\mathrm{Si} / \mathrm{Si}$ & $\mathrm{O}_{2}$ \\
\hline Control & No Aleatoria & $\mathrm{O}_{1}$ & $\mathrm{Si} / \mathrm{No}$ & $\mathrm{O}_{2}$ \\
\hline
\end{tabular}

Fuente: elaboración propia 


\section{Análisis de datos}

Se espera encontrar datos que apoyen la hipótesis por la cual, al exponer el grupo experimental a la experiencia con realidad virtual episódica, este experimentaría una disminución del consumo de agua significativamente, mayor que el grupo control. Para ello, se aplicará una ANOVA bifactorial de medidas repetidas a la disminución del consumo de agua. En la primera columna de la tabla 2 se muestra la reducción total del consumo, es decir, el cambio en la medida de consumo de agua tras la experiencia con realidad virtual y, en su caso, el carácter episódico de la misma. En la segunda y tercera columna se muestra la desviación típica y el nivel de significación de la reducción. Las otras tres columnas representan la diferencia media de reducción entre el grupo experimental y el grupo control, desviación típica y nivel de significación.

TABLA 2.

Diferencias intragrupo e intergrupo

\begin{tabular}{|c|c|c|c|c|c|c|}
\hline \multirow[b]{2}{*}{$\begin{array}{l}\text { Diferencia de } \\
\text { puntuación total }\end{array}$} & \multicolumn{3}{|c|}{ Diferencia intra-grupo } & \multicolumn{3}{|c|}{ Diferencia inter-grupo } \\
\hline & Media & $\begin{array}{l}\text { Desv. } \\
\text { T }\end{array}$ & $P$ & Media & Desv. T & $\mathrm{P}$ \\
\hline $\begin{array}{l}\text { G. Experimental } \\
\text { G. Control }\end{array}$ & $\begin{array}{l}M_{1} \\
M_{2}\end{array}$ & $\begin{array}{l}\mathrm{S}_{\mathrm{M} 1} \\
\mathrm{~S}_{\mathrm{M} 1} \\
\end{array}$ & $\begin{array}{l}\mathrm{p}_{1} \\
\mathrm{p}_{2}\end{array}$ & $\mathrm{M}_{12}$ & $\mathrm{~S}_{\mathrm{M} 12}$ & $\mathrm{p}_{12}$ \\
\hline
\end{tabular}

Fuente: elaboración propia

Se espera obtener una diferencia estadísticamente significativa $\mathrm{p}_{1}<0,05$ que apoye el hecho de que la exposición a las consecuencias futuras con realidad virtual episódica aumenta el consumo responsable de agua. Por otro lado, una diferencia estadísticamente significativa $\mathrm{p}_{12}<0,05$ nos sugeriría que esa mejora no es debida a la mera contemplación de efectos y recibir información sobre consumo responsable, sino a la exposición episódica, ya que en este caso se está comparando la mejora de las familias que han sido expuestas a la consecuencia futura episódica con aquellas que no.

\section{Discusión}

Se ha observado que las campañas generales de concienciación sobre el uso responsable del agua no son tan eficaces como otro tipo de campañas más personalizadas. Para entender por qué sucede esto y diseñar campañas más eficientes, se hace necesaria una mejor comprensión de las variables cognitivas que influyen en la actitud y el comportamiento de los individuos.

En este trabajo nos hemos centrado en la variable del descuento de la demora, que hemos considerado relevante si extrapolamos resultados positivos en otros estudios sobre otras conductas problemáticas, que se modifican mediante un cambio en esta variable. Además, el hecho de que los hogares de las regiones que han sufrido sequías y restricciones hacen un mejor uso del agua que aquellos que no han estado expuestos a estas, es un indicador de la importancia del descuento temporal o probabilístico en los comportamientos.

Se ha propuesto una campaña que lleve la realidad virtual a individuos o familias de distintas localidades y les ofrezcan vivir la experiencia de viajar al futuro y exponerse a una situación de sequía en primera persona. Se medirá el cambio de conducta en estas personas y se comparará con el de un grupo control. El análisis de los resultados, si son positivos, hará necesarios nuevos estudios donde se mida el descuento de la demora junto con otras variables cognitivas, para tratar de delimitar mejor el ámbito donde reside la posible causa cognitiva del efecto de cambio de conducta.

Como objetivo de este tipo de intervenciones, el comportamiento pro-ahorro y cambio de actitudes no sólo ha de influir sobre el ámbito en el consumo del hogar, también debe aspirar a lograr una generalización de los efectos en otros ámbitos como el exigir y apoyar un cierto tipo de políticas públicas o la inclusión de planes medioambientales en el marco de la responsabilidad social corporativa de las empresas que son clientes. 


\section{Referencias}

Ahn, S. J., Fox, J., Dale, K. R., y Avant, J. A. (2015). Framing virtual experiences: Effects on environmental efficacy and behavior over time. Communication Research, 42(6), 839-863. https://doi.org/10.1177/0093650214534973

Breckler, S. J. (1984). Empirical validation of affect, behavior, and cognition as distinct components of attitude. Journal of personality and social psychology, 47(6), 1191-1205. https://doi.org/10.1037//0022-3514.47.6.1191

Brick, C., y Lai, C. K. (2018). Explicit (but not implicit) environmentalist identity predicts pro-environmental behavior and policy preferences. Journal of Environmental Psychology, 58, 8-17. https://doi.org/10.1016/j.jenv p.2018.07.003

Collado, A., Johnson, P. S., Loya, J. M., Johnson, M. W., y Yi, R. (2017). Discounting of condom-protected sex as a measure of high risk for sexually transmitted infection among college students. Archives of sexual behavior, 46(7), 2187-2195. https://doi.org/10.1007/s10508-016-0836-x

Dharshing, S., y Hille, S. L. (2017). The energy paradox revisited: analyzing the role of individual differences and framing effects in information perception. Journal of Consumer Policy, 40(4), 485-508. https://doi.org/10.100 7/s10603-017-9361-0

European Enviroment Agency (EEA). (2001). Sustainable water use in Europe - Part 2: Demand management (Technical report no. 19), Copenhagen. Recuperado de https://www.eea.europa.eu/publications/Environment al_Issues_No_19

Gaudin, S. (2006). Effect of price information on residential water demand. Applied economics, 38(4), 383-393. http s://doi.org/10.1080/00036840500397499

Inman, D., y Jeffrey, P. (2006). A review of residential water conservation tool performance and influences on implementation effectiveness. Urban Water Journal, 3(3), 127-143. https://doi.org/10.1080/1573062060096 1288

Koffarnus, M. N., Jarmolowicz, D. P., Mueller, E. T., y Bickel, W. K. (2013). Changing delay discounting in the light of the competing neurobehavioral decision systems theory: a review. Journal of the experimental analysis of behavior, 99(1), 32-57. https://doi.org/10.1002/jeab.2

Lawyer, S. R., y Mahoney, C. T. (2017). Delay discounting and probability discounting, but not response inhibition, are associated with sexual risk taking in adults. The Journal of Sex Research, 55(7), 1-8. https://doi.org/10.108 $0 / 00224499.2017 .1350627$

Mellis, A. M., Snider, S. E., Deshpande, H. U., LaConte, S. M., y Bickel, W. K. (2019). Practicing prospection promotes patience: repeated episodic future thinking cumulatively reduces delay discounting. Drug and alcohol dependence, 204, 107507. https://doi.org/10.1016/j.drugalcdep.2019.06.010

Moglia, M., Cook, S., y Tapsuwan, S. (2018). Promoting Water Conservation: Where to from here? Water, 10(11), 1510. https://doi.org/10.3390/w10111510

Schacter, D. L., Benoit, R. G., y Szpunar, K. K. (2017). Episodic future thinking: Mechanisms and functions. Current opinion in behavioral sciences, 17, 41-50. https://doi.org/10.1016/j.cobeha.2017.06.002

Stavenhagen, M., Buurman, J., y Tortajada, C. (2018). Saving water in cities: Assessing policies for residential water demand management in four cities in Europe. Cities, 79, 187-195. https://doi.org/10.1016/j.cities.2018.03.008

Stein, J. S., Tegge, A. N., Turner, J. K., y Bickel, W. K. (2018). Episodic future thinking reduces delay discounting and cigarette demand: an investigation of the good-subject effect. Journal of behavioral medicine, 41(2), 269-276. $\mathrm{h}$ ttps://doi.org/10.1007/s10865-017-9908-1

Willis, R. M., Stewart, R. A., Panuwatwanich, K., Williams, P. R., y Hollingsworth, A. L. (2011). Quantifying the influence of environmental and water conservation attitudes on household end use water consumption. Journal of environmental management, 92(8), 1996-2009. https://doi.org/10.1016/j.jenvman.2011.03.023 


\section{Notas}

* Artículo de investigación.

Este artículo es resultado del trabajo realizado para el Congreso Nacional del Agua, sobre la Importancia de la telemetría, celebrado en Orihuela, España, en 2018.

\section{Licencia Creative Commons CC BY 4.0}

Cómo citar este artículo: Ochoa-Martínez, E., y Pérez-Gonzaga, S. (2019). Aumentar el consumo responsable de agua mediante la exposición a consecuencias futuras con realidad virtual. Ambiente $y$ Desarrollo, 23(45). https://doi.org/10.11144/Javeriana.ayd23-45.acra 